\title{
Stability of Naked Singularity arising in gravitational collapse of Type I matter fields
}

\author{
Sanjay B. Sarwe* \\ Department of Mathematics, S. F. S. College, \\ Seminary Hill, Nagpur-440 006, India \\ R. V. Saraykar ${ }^{\dagger}$ \\ Department of Mathematics, Nagpur University \\ Campus, Nagpur-440 033, India
}

\begin{abstract}
Considering gravitational collapse of Type I matter fields, we prove that, given an arbitrary $C^{2}$ - mass function $M(r, v)$ and a $C^{1}$ - function $h(r, v)$ (through the corresponding $C^{1}$ - metric function $\nu(t, r)$ ), there exist infinitely many choices of energy distribution function $b(r)$ such that the 'true' initial data $(M, h(r, v))$ leads the collapse to the formation of naked singularity. We further prove that the occurrence of such a naked singularity is stable with respect to small changes in the initial data. We remark that though the initial data leading to both black hole and naked singularity form a "big" subset of the true initial data set, their occurrence is not generic. The terms 'stability' and 'genericity' are appropriately defined following the theory of dynamical systems. The particular case of radial pressure $p_{r}(r)$ has been illustrated in details to get clear picture of how naked singularity is formed and how, it is stable with respect to initial data.
\end{abstract}

Keywords: Gravitational collapse, central-singularity, stability

PACS: 04.20.-q, 04.20.Dw, 04.40.Dg

\section{Introduction}

In recent papers $[1,2,3]$ Joshi, Dwivedi and Goswami have discussed the role of initial data in spherically symmetric gravitational collapse for Type I matter fields. It is shown that given the density $(\rho)$ and pressure profiles $\left(p_{r}, p_{\theta}\right)$ at the initial spacelike hypersurface from which the collapse evolves, there is a wide choice available to choose the velocity function and rest of the initial data so that the end state of collapse is either a black hole $(\mathrm{BH})$ or a naked singularity (NS). This result is significant for two reasons:- (1) it produces a

\footnotetext{
*email:sbsarwe_ngp@sancharnet.in

$\dagger$ †email:sarayaka_ngp@sancharnet.in
} 
substantially "big" initial data set which under gravitational collapse results into a naked singularity. (2) Type I matter fields include most of the known physical forms of matter like dust, perfect fluid. At least in spherically symmetric case, this result shows that while considering black hole physics, one must discard certain part of initial data set that leads the collapse to a naked singularity. However, all of the initial data is not independent, so it poses a question, which initial data set shall evolve into a NS as a result of gravitational collapse? Further, having a NS for certain initial data set is not enough because if it looses its characteristic for small perturbation in the neighbourhood of that certain initial data set then the NS is not serious enough to challenge cosmic censorship conjecture [4]. Therefore, the all important question ie. is the NS developed from certain initial data set a stable one?

This motivates us to study both the above mentioned questions. We prove that given a $C^{2}$-mass function $M(t, r)$ and $C^{1}$ - velocity function $\nu(t, r)$ on an initial spacelike hypersurface, there always exists a $C^{1}$ - function $b(r)$ such that the total initial data comprising of mass function (includes density and pressures), a function $\nu(r)$ and energy distribution function $b(r)$ evolves into a naked singularity as a result of gravitational collapse.

F.I. Cooperstock and et al. [5] have shown that if the radial pressure $p_{r}$ is positive , $r>0$ and having strictly positive mass function then non-central singularity is covered by the horizon irrespective of the sign of tangential pressure $p_{\theta}$. The stability of central shellfocusing singularity at $r=0$ that we are discussing in this paper is with respect to small changes in the initial data comprising of mass function and velocity function. "Small" changes in the initial data are with respect to appropriate norm on the the functions space of all physically reasonable initial data. This concept of stability is analogous to that of structural stability in the theory of dynamical systems and is explained in section 4. Essentially, we prove that the set of initial data leading the collapse to central shell focusing naked singularity is an open subset of the set of all initial data in a suitable functions space. That we get a covered singularity which is non-central when $p_{r}>0$ does not disturb the stability of central shell focusing singularity (naked or covered) with respect to small changes in initial data.

In section 2, we briefly summarize analysis given in [3] and state the conditions on the initial data under which the collapse will lead to a naked singularity. In section 3, we analyze the condition of existence of naked singularity to prove our assertion mentioned above by using existence theory of first order ordinary differential equations. Thereafter, in section 4, we define and explain the concept of stability and genericity that we follow in this paper and we prove openness of initial data set. We, then, discuss genericity of this set. In section 5, we study the particular case $p_{r}(r)$ to demonstrate the involvement of $p_{r}$ and $p_{\theta}$ as an initial data in the occurrence of NS, its stability and non-generic nature. Finally, we make some concluding remarks.

\section{Field equations, initial data and naked singularity}

In this section, we summarize analysis from [3]. The general spherically symmetric metric describing space-time geometry within the collapsing cloud can be described in comoving 
coordinates $(t, r, \theta, \phi)$ by

$$
d s^{2}=-e^{2 \nu(t, r)} d t^{2}+e^{2 \psi(t, r)} d r^{2}+R^{2}(t, r) d \Omega^{2}
$$

where $d \Omega^{2}=d \theta^{2}+\sin ^{2} \theta d \phi^{2}$ is the metric on an 2-sphere. The stress-energy tensor for Type I field in a diagonal form is given by [6]

$$
T_{t}^{t}=-\rho, T_{r}^{r}=p_{r}, T_{\theta}^{\theta}=T_{\phi}^{\phi}=p_{\theta}
$$

The quantities $\rho, p_{r}$ and $p_{\theta}$ are the energy density, radial and tangential pressures respectively. We take the matter field to satisfy weak energy condition i.e. the energy density measured by any local observer be non-negative, so for any vector $V^{i}$, we must have, $T_{i k} V^{i} V^{k} \geq 0$ which means $\rho \geq 0 ; \rho+p_{r} \geq 0 ; \rho+p_{\theta} \geq 0$.

Einstein field equations for the metric (1) are

$$
\begin{aligned}
& \rho=\frac{F^{\prime}}{R^{2} R^{\prime}} \\
& p_{r}=-\frac{\dot{F}}{R^{2} \dot{R}} \\
& \nu^{\prime}=\frac{2\left(p_{\theta}-p_{r}\right)}{\rho+p_{r}} \frac{R^{\prime}}{R}-\frac{p_{r}^{\prime}}{\rho+p_{r}} \\
&-2 \dot{R}^{\prime}+R^{\prime} \frac{\dot{G}}{G}+\dot{R} \frac{H^{\prime}}{H}=0 \\
& G-H=1-\frac{F}{R}
\end{aligned}
$$

where we have defined $G(t, r)=e^{-2 \psi}\left(R^{\prime}\right)^{2}$ and $H(t, r)=e^{-2 \nu}(\dot{R})^{2}$.

The total mass in a shell of collapsing cloud of comoving radius $r$ is represented by the arbitrary function $F=F(t, r)$. The weak energy conditions imply $F \geq 0$. The regularity at the initial epoch $t=t_{i}$ is preserved by $F\left(t_{i}, 0\right)=0$ i.e. the mass function should vanish at the centre of the cloud. The density singularity in the space-time is at $R=0$ and $R^{\prime}=0$, the later one is due to shell-crossings and can possibly be removed from the space-time [7], so we consider here only a physical singularity where all matter shells collapse to a zero physical radius known as shell-focusing singularity. We can use scaling freedom available for the radial co-ordinate $r$ to introduce the function $v(t, r)$ by the relation

$$
R(t, r)=r v(t, r),
$$

we have $v\left(t_{i}, r\right)=1 ; v\left(t_{s}(r), r\right)=0$ and for collapse $\dot{v}<0$. Above relation is obtained by defining $v(t, r)=R / r$ [8]. The time $t=t_{s}(r)$ corresponds to the shell-focusing singularity $R=0$. The six arbitrary functions of the shell radius $r$ as given by $\nu\left(t_{i}, r\right)=\nu_{o}(r)$, $\psi\left(t_{i}, r\right)=\psi_{o}(r), \quad R\left(t_{i}, r\right)=r, \quad \rho\left(t_{i}, r\right)=\rho_{o}(r), \quad p_{r}\left(t_{i}, r\right)=p_{r_{o}}(r), \quad p_{\theta}\left(t_{i}, r\right)=p_{\theta_{o}}(r)$ evolve the dynamics of the initial data prescribed at the initial epoch $t=t_{i}$. From equation (5), we obtain

$$
\nu_{o}(r)=\int_{0}^{r}\left(\frac{2\left(p_{\theta_{o}}-p_{r_{o}}\right)}{r\left(\rho_{o}+p_{r_{o}}\right)}-\frac{p_{r_{o}}^{\prime}}{\rho_{o}+p_{r_{o}}}\right) d r
$$


which indicates that not all the above initial data are mutually independent and that $\nu_{o}(r)$ has the form,

$$
\nu_{o}(r)=r^{2} g(r)
$$

where $g(r)$ is at least a $C^{1}$ function of $r$ for $r=0$, and at least a $C^{2}$ function for $r>0$. Let us now assume the physically reasonable behaviour of the initial data at the center $r=0$ i.e. $p_{r_{o}}^{\prime}(0)=p_{\theta_{o}}^{\prime}(0)=0$ and $p_{r_{o}}(0)-p_{\theta_{o}}(0)=0$. For details, we refer [1]. We have a total of five equations with seven unknowns, namely $\rho, p_{r}, p_{\theta}, \nu, \psi, R$ and $F$ giving us the freedom of choice of two functions. Selection of these two free functions, subject to the given initial data and the weak energy condition above, determines the matter distribution and the metric of the space-time and thus, leads to a particular time evolution of the initial data. The existence and uniqueness of solution of the system of field equations with above mentioned initial data has been discussed by Joshi and Dwivedi [9, 1]. The solution continues to exist in the neighbourhood of the singularity given by $R=0$. A general mass function for the cloud can be considered as

$$
F(t, r)=r^{3} M(r, v)
$$

where $M(r, v)$ is regular and continuously twice differentiable. Using equation (11) in equations (3) and (4), we obtain

$$
\rho=\frac{3 M+r\left[M_{, r}+M_{, v} v^{\prime}\right]}{v^{2}\left(v+r v^{\prime}\right)} ; \quad p_{r}=-\frac{M_{, v}}{v^{2}}
$$

Then as $v \rightarrow 0, \rho \rightarrow \infty$ and $p_{r} \rightarrow \infty$ i.e. both the density and radial pressure blow up at the singularity. The regular density distribution at the initial epoch is given by $\rho_{o}(r)=3 M(r, 1)+r M(r, 1), r$.

We take the initial surface to be the cloud given by $0 \leq r \leq r_{c}$ for some finite $r_{c}$ on which the initial data, namely the mass function $F(t, r)$, the metric function $\nu(t, r)$ and the function $b(r)$ ( to follow) evolve as the collapse begins according to the Type I field equations. So, we take $M(r, v)>0$ and at least $C^{2}$ in $\mathcal{D} \equiv\left[0, r_{c}\right] \times[0,1]$, for varying $r \in\left[0, r_{c}\right], v \in[0,1]$. Consider the general metric function,

$$
\nu(t, r)=A(t, R)
$$

where $A(t, R)$ is arbitrary, continuously differentiable function of $t$ and $R$, with the initial constraint $A\left(t_{i}, R\right)=\nu_{o}(r)$. We use equation (13) in equation (5) to get

$$
2 p_{\theta}=R A_{, R}\left(\rho+p_{r}\right)+2 p_{r}+\frac{R p_{r}^{\prime}}{R^{\prime}}
$$

From above equation, we conclude that the tangential pressure also blows up at the singularity. Also, the use of equation (13) in equation (6), yields

$$
G(t, r)=d(r) e^{2\left(A-\int A_{, t} d t\right)}
$$

where $d(r)$ is another arbitrary continuously differentiable function of $r$. From equation (10), we generalize the form of $A(t, R)$ as $A(t, R)=r^{2} g_{1}(r, v)$, where $g_{1}(r, v)$ is a continuously differentiable function and $g_{1}(r, 1)=g(r)$. Similarly, we have $A-\int A_{, t} d t=r^{2} g_{2}(r, v)$ and at the initial epoch $g_{2}(r, 1)=g(r)$. We write

$$
d(r)=1+r^{2} b(r) .
$$


where $\mathrm{b}(\mathrm{r})$ is the energy distribution function for the collapsing shells. Then using equations $(11),(13)$ and (15) in equation (7), we get

$$
\sqrt{R} \dot{R}=-e^{r^{2} g_{1}(r, v)} \sqrt{\left[1+r^{2} b(r)\right] R e^{r^{2} g_{2}(r, v)}-R+r^{3} M}
$$

where negative sign is chosen since, for the collapse, $\dot{R}<0$. Defining a function $h(r, v)$ as

$$
h(r, v)=\frac{e^{r^{2} g_{2}(r, v)}-1}{r^{2}}=2 g_{2}(r, v)+O\left(r^{2} v^{2}\right)
$$

and substituting equation (18) in equation (17), we get

$$
\sqrt{v} \dot{v}=-\sqrt{e^{2 r^{2}\left(g_{1}+g_{2}\right)} v b(r)+e^{2 r^{2} g_{1}}(v h+M)}
$$

Integrating the above equation, we have

$$
t(v, r)=\int_{v}^{1} \frac{\sqrt{v} d v}{\sqrt{e^{2 r^{2}\left(g_{1}+g_{2}\right)} v b(r)+e^{2 r^{2} g_{1}(v h+M)}}}
$$

Here, the variable $r$ is treated as a constant in the above equation. Expanding $t(v, r)$ around the centre, we get

$$
t(v, r)=t(v, 0)+r \chi(v)+O\left(r^{2}\right)
$$

where the function

$$
\chi(v)=-\frac{1}{2} \int_{v}^{1} \frac{\sqrt{v}\left[v b^{\prime}(0)+v h_{, r}(0, v)+M_{, r}(0, v)\right]}{[v b(0)+v h(0, v)+M(0, v)]^{3 / 2}} d v .
$$

Now, it is clearly seen that the value of $\chi(0)$ depends on the functions $b(0), M(0, v)$ and $h(0, v)$, which in turn, depend on the initial data at the initial surface $t=t_{i}$. Thus, a tangent to the singularity curve $t=t_{s_{o}}$ is completely determined by the given set of density, pressure, velocity function $\nu$ and function $b(r)$. Further, from equation (19), we can write

$$
\sqrt{v} v^{\prime}=\chi(v) \sqrt{v b(0)+v h(0, v)+M(0, v)}+O\left(r^{2}\right)
$$

If the neighbourhood of the centre $R=0, r=0$ gets trapped earlier than the formation of singularity, then it is covered ( i.e. occurrence of a black hole), and if otherwise happens the singularity is naked (ie. singularity can be observed locally or by a faraway observer) [7]. For examination of the nature of central singularity at $R=0, r=0$, we consider the equation for outgoing radial null geodesic, $d t / d r=e^{(\psi-\nu)}$ and test, if there would be any families of null geodesics coming out of the singularity. Further, we write the null geodesic equation in terms of the variables $\left(u=r^{\alpha}, R\right)$, choosing $(\alpha=5 / 3)$, and using equation (7), we obtain

$$
\frac{d R}{d u}=\frac{3}{5}\left(\frac{R}{u}+\frac{\sqrt{v} v^{\prime}}{\sqrt{\frac{R}{u}}}\right)\left(\frac{1-\frac{F}{R}}{\sqrt{G}[\sqrt{G}+\sqrt{H}]}\right)
$$

If the null geodesics terminate at the singularity in the past with a definite tangent, then at the singularity, we have $d R / d u>0$, in the $(u, R)$ plane with a finite value. Hence, all 
points $r>0$ on the singularity curve are covered since $F / R \rightarrow \infty$ with $d R / d u \rightarrow-\infty$ and only the singularity at the centre $r=0$ could be naked. For the case, when $R^{\prime}>0$ near the central singularity, we have

$$
x_{o}=\lim _{t \rightarrow t_{s}} \lim _{r \rightarrow o} \frac{R}{u}=\left.\frac{d R}{d u}\right|_{t \rightarrow t_{s}, r \rightarrow o}
$$

and use of equations (23) and (24) yield $x_{o}{ }^{3 / 2}=(3 / 2) \sqrt{M(0,0)} \chi(0)$ and the radial null geodesic emerging from the singularity in $(R, u)$ co-ordinates is $R=x_{o} u$, or in $(t, r)$ plane, it is given by $t-t_{s}(0)=x_{o} r^{5 / 3}$. Therefore, if $\chi(0)>0$ then $x_{o}>0$, and we get radially outgoing null geodesics coming out from the singularity, giving rise to a naked central singularity. However, if $\chi(0)<0$ then we have a black hole solution, as there will be no such trajectories coming out. For $\chi(0)=0$, we will have to take into account the next higher order non-zero term in the singularity curve equation, and a similar analysis can be carried out by choosing a different value of $\alpha$.

Thus, it is clearly seen as to how the initial data determines the NS/BH phases as end states of collapse, since $\chi(0)$ is determined by these initial profiles for the collapsing matter given by equation (22).

\section{Existence of energy distribution function $b(r)$ lead- ing to NS}

In this section, we prove the assertion mentioned in the introduction. We choose $b(r)$ to satisfy the differential equation on a constant v-surface

$$
\frac{1}{2} \frac{\sqrt{v}\left[v b^{\prime}(r)+v h_{, r}(r, v)+M_{, r}(r, v)\right]}{[v b(r)+v h(r, v)+M(r, v)]^{3 / 2}}=B(r, v)
$$

for $0 \leq r \leq r_{c}$ where $B(r, v)$ is a continuous function defined on $\mathcal{D}$ such that

$$
B(0, v)=\frac{1}{2} \frac{\sqrt{v}\left[b^{\prime}(0) v+v h_{, r}(0, v)+M_{, r}(0, v)\right]}{[v b(0)+v h(0, v)+M(0, v)]^{3 / 2}}<0
$$

for all $v$ in $[0,1]$. It will then follow that

$$
\chi(0)=\lim _{v \rightarrow 0} \chi(v)=-\int_{0}^{1} B(0, v) d v>0 .
$$

This condition ensures that central shell-focusing singularity will be naked.

We, now, discuss the existence of $b(r)$ as a solution of the differential equation (26). We put

$$
x(r, v)=v b(r)+v h(r, v)+M(r, v)
$$

a continuous function of $r$, in equation (26) and rearranging it, we get

$$
\frac{d x}{d r}=\frac{1}{\sqrt{v}}\left[2 B(r, v) x^{3 / 2}\right] \equiv f(x, r)
$$


with the initial condition

$$
x(0, v)=v b(0)+v h(0, v)+M(0, v) .
$$

Let us ensure the existence of $C^{1}$ - function $x(r, v)$ as a solution of above initial value problem defined throughout the cloud. The function $f(x, r)$ is continuous in $r$, with $x$ restricted to a bounded domain. With such domain of $r$ and $x, f(x, r)$ is also $C^{1}$ - function in $x$ which means $f(x, r)$ is Lipschitz continuous in $x$. Therefore, the differential equation (30) has a unique solution satisfying initial condition (31).

Further, we can ensure that the solution will be defined over the entire cloud i.e. for all $r$ in $\left[0, r_{c}\right]$ by using the freedom in the choice of arbitrary function $B(r, v)$. For this , we consider the domain $\left[0, r_{c}\right] \times[0, d]$ for some finite $d$. Let $S$ be the supremum of the modulus of $f(x, r)$. Then the differential equation (30) has a unique solution defined over the entire cloud provided [10]

$$
r_{c} \leq \inf \left(r_{c}, \frac{d}{S}\right)=\frac{d}{S}
$$

This yields

$$
\max _{0 \leq r \leq r_{c}, 0 \leq x \leq d}\left|\frac{1}{\sqrt{v}}\left[2 B(r, v) x^{3 / 2}\right]\right| \leq \frac{d}{r_{c}} .
$$

Condition (32), in turn, will be satisfied if the weaker condition

$$
0 \leq|B(r, v)| x^{3 / 2} \leq \frac{d \sqrt{v}}{2 r_{c}}
$$

holds for all $r$ in $\left[0, r_{c}\right]$.

The collapsing cloud may start with $r_{c}$ small enough so that the expression $d \sqrt{v} / 2 r_{c}$ which is always positive, satisfies the condition (34) with $x$ restricted to a bounded domain. We then have infinitely many choices for the function $B(r, v)$, which is continuous and satisfies conditions (27) and (34) for each choice of $v$. For each such $B(r, v)$, there will be a unique solution $x(r, v)$ of the differential equation (30), satisfying initial condition (31), defined over the entire cloud and in turn, there exist a unique function $b(r)$ for each such choice of $B(r, v)$, that is given by the expression

$$
b(r)=\frac{x(r, v)-M(r, v)-v h(r, v)}{v}
$$

over $\left[0, r_{c}\right]$. Thus, for a given constant $v$-surface and given initial data of mass function $F(t, r)=r^{3} M(r, v)$ and $h(r, v)=\left(e^{r^{2} g_{2}(r, v)}-1\right) / r^{2}$ satisfying physically reasonable conditions (expressed on $M$ ), there exists infinitely many choices for the function $b(r)$ such that condition (27) is satisfied. The condition continues to hold as $v \rightarrow 0$, because of continuity. Hence, the central singularity developed in the collapse is a naked singularity. 


\section{Definition of stability and genericity of NS/BH}

In this section, we give the appropriate definitions of stability and genericity. Our definitions of stability and genericity are based on the structural stability of a dynamical system, and genericity of a property of a dynamical system respectively. For more details, refer chapter 7, §3,4 of Abraham and Marsden [11].

Let $\mathbf{M}$ be a manifold on which a vector field or a dynamical system is defined. Let $\mathcal{X}(\mathbf{M})$ be the space of all vector fields on $\mathbf{M}$. Whitney $C^{r}$-topology on $\mathcal{X}(\mathbf{M})$, generated by the norm is given by

$$
\|f\|_{r}=\sup \left\{\sum_{k=0}^{r}\left\|D^{k} f(u)\right\| / u \in U\right\},
$$

where $V$ and $W$ are vector spaces, $U$ an open subset of $V$, and $f: U \rightarrow W$. $D^{k} f$ denotes $k$ th Frechet derivative of $f . \mathcal{X}(\mathbf{M})$ endowed with Whiteny $C^{r}$ - topology is denoted by $\mathcal{X}^{r}(\mathbf{M})$.

Let $X_{1}$ be a vector field or a dynamical system on $\mathbf{M}$. Then $X_{1}$ is structurally stable if there is a neighbourhood $\Phi$ of $X_{1} \in \mathcal{X}^{r}(\mathbf{M})$ in the Whitney $C^{r}$ - topology such that $Y_{1} \in \Phi$ implies $X_{1}$ and $Y_{1}$ are topologically conjugate i.e. they have equivalent phase portraits. This means that there is a homeomorphism $h_{o}: \mathbf{M} \rightarrow \mathbf{M}$ carrying oriented orbits of $X_{1}$ to oriented orbits of $Y_{1}$.

A property of vector fields in $\mathcal{X}^{r}(\mathbf{M})$ is a proposition $P\left(x_{1}\right)$ with a variable $x_{1} \in$ $\mathcal{X}^{r}(\mathbf{M})$. A property $P\left(x_{1}\right)$ with a variable $x_{1} \in \mathcal{X}^{r}(\mathbf{M})$ is generic if the subset $\left\{x_{1} \in\right.$ $\left.\mathcal{X}^{r}(\mathbf{M}) / P\left(x_{1}\right)\right\} \subset \mathcal{X}^{r}(\mathbf{M})$ contains a residual set.

A subset $A$ of a topological space $X_{1}$ is called residual if and only if $A$ is the intersection of a countable family of open dense subsets of $X_{1}$. A topological space $X_{1}$ is a Baire space if and only if every residual set is dense. We also know that every complete metric space and in particular every Banach space is a Baire space. Also, whether $\mathbf{M}$ is compact or not, $\mathcal{X}^{r}(\mathbf{M})$ is a Baire space.

In our case, we apply these definitions analogously to the evolution of initial data into a gravitational collapse leading to a naked singularity or a black hole. We treat evolving initial data as a vector field or a dynamical system and consider the space of all initial data with sufficient differentiability defined on a collapsing compact spherical shell of Type I field, in place of $\mathcal{X}(\mathbf{M})$, endowed with a suitable $C^{r}$-topology. Property $P$ of a dynamical system becomes the property of initial data, namely, whether this initial data leads the collapse to a naked singularity or a black hole. Thus, the definitions of stability of a naked singularity and genericity of its occurrence can be stated as follows.

Let $I_{o}$ be the initial data set, which when evolves, leads the collapse to a NS/BH. We say that a NS/BH is stable, if there is a neighbourhood I of $I_{o}$ in $C^{r}$-topology such that, if $I_{1}$ is another initial data in I, then $I_{1}$ also leads the collapse to a NS/BH. In other words, if the set of initial data leading the collapse to a NS/BH forms an open subset of $C^{r}$-space of all initial data, then the NS/BH will be stable. Similarly, occurrence of a NS/BH will be said to be generic, if the set of all initial data leading to NS/BH, is a dense subset of the parent $C^{r}$-space. All sets of initial data are required to satisfy the constraint equations, in addition to the energy conditions. 


\subsection{Stability of Naked Singularity}

The analysis in section III shows that the only conditions on the initial data which evolves the collapse into a naked singularity are the energy conditions. Hence, for stability, we have to examine energy conditions only. What we now show is that, the set of such initial data satisfy energy conditions forms an open subset of the space of all initial data. For this , we use the technique of Saraykar and Ghate [12]. The energy conditions $\rho \geq 0 ; \rho+p_{r} \geq 0$; $\rho+p_{\theta} \geq 0$ can be written in the form

$$
\begin{array}{r}
{\left[3 M+r M_{, r}+r v^{\prime} M_{, v}\right] \equiv E_{1} \geq 0, \quad\left[3 M+r M_{, r}-v M_{, v}\right] \equiv E_{2}>0} \\
{\left[3 w M+r w M_{, r}+w_{1} M_{, v}-r v\left(v+r v^{\prime}\right) M_{, r v}\right] \equiv E_{3} \geq 0}
\end{array}
$$

where $w=2\left(v+r v^{\prime}\right)+r v \nu_{, r}$ and $w_{1}=2 r^{2} v^{\prime 2}-(2+r) v^{2} \nu_{, r}$ on the domain $\mathcal{D}$. Here, we have used an assumption that $v$ is an increasing function of $r$ so that $v^{\prime} \geq 0$ on any surface $t=t_{j}$ say, so that no shell-crossing singularity condition $\left(i e . R^{\prime}>0\right)$ holds. So, this is a valid assumption. We know that as the nuclear fuel exhausts in a star, it starts collapsing under its tremendous gravitational pull, and in this case, in the formation of central singularity radial pressure diverges, so we assume radial pressure $p_{r}$ to be non-negative throughout the gravitational collapse. Therefore, from equation (12), we have $M_{v} \leq 0$. Then, $E_{1} \leq E_{2}$. Hence, only $E_{1}$ and $E_{3}$ shall take part in further stability analysis.

We assume that $X$ be an infinite dimensional Banach space of all $C^{2}$ real-valued functions defined on $\mathcal{D}$, endowed with the norms

$$
\begin{gathered}
\|M(r, v)\|_{1}=\sup _{\mathcal{D}}|M|+\sup _{\mathcal{D}}\left|M_{, r}\right|+\sup _{\mathcal{D}}\left|M_{, v}\right| \\
\text { and }\|M(r, v)\|_{2}=\sup _{\mathcal{D}}|M|+\sup _{\mathcal{D}}\left|M_{, r r}\right|+\sup _{\mathcal{D}}\left|M_{, r v}\right|+\sup _{\mathcal{D}}\left|M_{, v v}\right|
\end{gathered}
$$

These norms are equivalent to the standard $C^{1}$ and $C^{2}$ norms

$$
\begin{gathered}
\|M(r, v)\|_{1}=\sup _{\mathcal{D}}\left(|M|+\left|M_{, r}\right|+\left|M_{, v}\right|\right) \\
\text { and }\|M(r, v)\|_{2}=\sup _{\mathcal{D}}\left(|M|+\left|M_{, r r}\right|+\left|M_{, r v}\right|+\left|M_{, v v}\right|\right)
\end{gathered}
$$

Let $\mathcal{G}=\left\{M(r, v): M>0, M\right.$ is $C^{2}, E_{1}>0$ and $E_{3}>0$ on $\left.\mathcal{D}\right\}$ be a subset of $X$.

We show that $\mathcal{G}$ is an open subset of $X$. For $M$ in $\mathcal{G}$, let us put $\delta=\min (M), \gamma=\min \left(E_{1}\right)$ $, \beta=\min \left(E_{3}\right), \lambda=\max (w), \lambda_{1}=\max \left(w_{1}\right), \lambda_{2}=\max \left(v+r v^{\prime}\right)$ and $\lambda_{3}=\max \left(v^{\prime}\right)$ for varying $r$ in $\left[0, r_{c}\right]$ and $v \epsilon[0,1]$, the functions involved herein are all continuous functions defined on a compact domain $\mathcal{D}$ and hence, their maxima and minima exist. We define a positive real number

$$
\mu=\frac{1}{2} \min \left\{\delta, \frac{\gamma}{9}, \frac{\gamma}{3 r_{c}}, \frac{\gamma}{3 r_{c} \lambda_{3}}, \frac{\beta}{12 \lambda}, \frac{\beta}{4 r_{c} \lambda}, \frac{\beta}{4 \lambda_{1}}, \frac{\beta}{4 r_{c} \lambda_{2}}\right\}
$$

Let $M_{1}(r, v)$ be $C^{2}$ in $\mathcal{D}$ with $\left\|M-M_{1}\right\|_{1}<\mu$ and $\left\|M-M_{1}\right\|_{2}<\mu$. Using definition (36), we get $\left|M_{1}-M\right|<\mu,\left|M_{1, r}-M_{, r}\right|<\mu,\left|M_{1, v}-M_{, v}\right|<\mu$ and $\left|M_{1, r v}-M_{, r v}\right|<\mu$ over $\mathcal{D}$. Therefore, for choice of $\mu$, the respective inequalities are

$$
M_{1}>M-\frac{\delta}{2}>0, \quad 3\left|M_{1}-M\right|<\frac{\gamma}{6},
$$




$$
\begin{array}{rr}
r\left|M_{1, r}-M_{, r}\right| \leq r_{c}\left|M_{1, r}-M_{, r}\right|<\frac{\gamma}{6}, & r v^{\prime}\left|M_{1, v}-M_{, v}\right| \leq r_{c} \lambda_{3}\left|M_{1, v}-M_{, v}\right|<\frac{\gamma}{6}, \\
3 w\left|M_{1}-M\right|<\frac{\beta}{8}, & r w\left|M_{1, r}-M_{, r}\right| \leq r_{c} \lambda\left|M_{1, r}-M_{, r}\right|<\frac{\beta}{8}, \\
w_{1}\left|M_{1, v}-M_{, v}\right| \leq r_{c} \lambda_{1}\left|M_{1, v}-M_{, v}\right|<\frac{\beta}{8}, & r v\left(v+r v^{\prime}\right)\left|M_{1, r v}-M_{, r v}\right|<\frac{\beta}{8}
\end{array}
$$

that are satisfied on $\mathcal{D}$. The $I I^{\text {nd }}, I I I^{r d}$ and $I V^{\text {th }}$ inequations from above yield

$$
3\left|M_{1}-M\right|+r\left|M_{1, r}-M_{, r}\right|+r v^{\prime}\left|M_{1, v}-M_{, v}\right|<\frac{\gamma}{2}<\gamma \leq E_{1}
$$

Further, we can write $\quad\left|\left[3 M_{1}+r M_{1, r}+r v^{\prime} M_{1, v}\right]-E_{1}\right|<E_{1}$ where $E_{1}>0$ on $\mathcal{D}$. Hence, $\left[3 M_{1}+r M_{1, r}+r v^{\prime} M_{1, v}\right]>0$ on $\mathcal{D}$. Using similar analysis for last four inequations of equation (37), we obtain $\left[3 w M_{1}+r w M_{1, r}+w_{1} M_{1, v}-r v\left(v+r v^{\prime}\right) M_{1, r v}\right]>0$ on $\mathcal{D}$ provided $w>0$ and $w_{1}>0$ on $\mathcal{D}$.

Thus, $M_{1}>0, M_{1}$ is $C^{2},\left[3 M_{1}+r M_{1, r}+r v^{\prime} M_{1, v}\right]>0$ and $\left[3 w M_{1}+r w M_{1, r}+w_{1} M_{1, v}-\right.$ $\left.r v\left(v+r v^{\prime}\right) M_{1, r v}\right]>0$ on $\mathcal{D}$ provided $w>0$ and $w_{1}>0$ throughout $\mathcal{D}$. Therefore, $M_{1}(r, v)$ also lies in $\mathcal{G}$ and hence, $\mathcal{G}$ is an open subset of $X$.

The conditions $w>0$ and $w_{1}>0$ required for openness of $\mathcal{G}$ on $\mathcal{D}$, impose conditions on the function $\nu(t, r), \nu(t, r)=A(t, R)$ and $A-\int A_{, t} d t=r^{2} g_{2}(r, v)$, therefore, $h(r, v)=$ $e^{r^{2} g_{2}(r, v)-1} / r^{2}$ is constrained by the conditions $w>0$ and $w_{1}>0$ in domain $\mathcal{D}$. Now, let $Y$ be an infinite dimensional Banach space of all $C^{1}$ real-valued functions defined on $\mathcal{D}$. The set $\mathcal{H}$ of functions $h(r, v)$ with restrictions $w>0$ and $w_{1}>0$ would form (by similar technique) an open subset of $Y$. Then $\mathcal{G} \times \mathcal{H}$ is an open subset in the product space $X \times Y$.

Taking $\left(M_{1}, h_{1}(r, v)\right)$ in the neighbourhood of $(M, h(r, v))$ in $G \times \mathcal{H}$, and using equation (34) analogously for $M_{1}$ and $h_{1}$, we have a choice of infinitely many $B_{1}(r, v)$, such that for each such $B_{1}(r, v)$, there will exist a unique $b_{1}(r)$ so that the initial data of mass function $r^{3} M_{1}$ and $h_{1}(r, v)$ together will lead the collapse to formation of a naked singularity. Thus, naked singularity arising from $(M, h(r, v))$ is $C^{2}$ - stable in the sense defined above.

The analysis given above, guarantee the existence of a metric function $\nu(t, r)$ for a given initial data set. Such choice of $\nu(t, r)$ and expressions for $G$ and $H$ together will yield the metric (1) as an exact solution leading to the occurrence of naked singularity. Thus, given initial data and the weak energy conditions above determine the matter distribution and the metric of the space-time.

\subsection{Genericity of NS/BH}

Let us discuss the genericity of the occurrence of these singularities. We have seen that in Type I matter field collapse, the end state of collapse is governed by the choice of initial data. As explained above, occurrence of a NS is generic if the set $\mathcal{G} \times \mathcal{H}$ of 'true' initial data $(M, \mathrm{~h}(\mathrm{r}, \mathrm{v}))$, contains a residual set in the space. The space $X \times Y$ contains negative functions which cannot be realized as a limit of a sequence of positive functions belonging to $\mathcal{G} \times \mathcal{H}$. Hence, $\mathcal{G} \times \mathcal{H}$ is not dense in $X \times Y$. The space under consideration is a Banach space. Every complete metric space and in particular every Banach space is a Baire space, and since in a Baire space, every residual set is dense, it follows that $\mathcal{G} \times \mathcal{H}$ is not residual. Therefore, we conclude that occurrence of naked singularities is not $C^{2}$-generic. Further, since the set $\mathcal{G} \times \mathcal{H}$ is open, it cannot be nowhere dense and hence $\mathcal{G} \times \mathcal{H}$ is not a meagre set either. Thus, it is a substantially "big" set. 


\section{$5 \quad$ Special case $p_{r}=p_{r}(r)$}

The case $p_{r}=1$ has been studied by Goswami and Joshi and it is shown that the presence of a non-vanishing pressure gradient gives rise to either formation of NS or a $\mathrm{BH}$ [13]. We illustrate above analysis by studying a special case where $p_{r}$ be a function of $r$ alone. It gives a more clear picture of the occurrence of naked singularity and its stability. We choose two allowed free functions $p_{r}$ and $\nu(t, r)$ as follows:

$$
p_{r}=p_{r}(r), \quad \nu(t, r)=c(t)+\eta(R)
$$

Now, let us see, how the Einstein's field equations react to this choice which will decide the evolution of collapse. As $p_{r}$ is a function $r$ alone, integrating equation (4), we obtain

$$
F(t, r)=-\frac{p_{r}}{3} R^{3}+z(r)
$$

where $z(r)$ is another arbitrary function of $r$. We need to choose $z(r), z(r)=\frac{2}{3} r^{3} p_{r}(r)$ so that the mass function takes the form

$$
F=\frac{p_{r}}{3}\left(2 r^{3}-R^{3}\right)
$$

that satisfies the regularity conditions at the initial epoch $F\left(t_{i}, r\right)=p_{r} r^{3} / 3$ and $F\left(t_{i}, 0\right)=$ 0 . Therefore, $F \geq 0$ provided $p_{r} \geq 0$. Hence, non-negativity of radial pressure shall maintain non-negativity of mass function throughout the gravitational collapse. Next, equation (3) becomes

$$
\rho=\frac{1}{3} \frac{3 p_{r}\left(2 r^{2}-R^{2} R^{\prime}\right)+p_{r}^{\prime}\left(2 r^{3}-R^{3}\right)}{R^{2} R^{\prime}}
$$

The density blows up at the singularity in the limit of approach to the singularity ie. as $r \rightarrow 0$ and $t \rightarrow t_{s}$. At the initial epoch $t=t_{i}$,

$$
\rho=p_{r}+\frac{1}{3} r p_{r}^{\prime} \geq 0
$$

, an all important relation between energy density and radial pressure at the initial epoch. Equation (6 ) takes the form

$$
G=e^{2 \eta(R)} d_{p}(r)
$$

with $d_{p}(r)=1+r^{2} b_{p}(r)$ where $b_{p}$ is the energy distribution function for the collapsing shells. Using equation (38) in equation (5), we obtain

$$
\left(\rho+p_{r}\right) \eta_{, R} R^{\prime} R=2\left(p_{\theta}-p_{r}\right) R^{\prime}-R p_{r}^{\prime}
$$

Since the density blows up at the singularity, from above equation, it is clear that the tangential pressure also blows up in the limit of approach to the singularity. Further, we find that all of initial data is not independent at the initial epoch $t=t_{i}$

$$
\left(\rho+p_{r}\right) \eta_{, R} r=2\left(p_{\theta}-p_{r}\right)-r p_{r}^{\prime} .
$$

Above equation and equation (42) suggest that $b_{p}$, radial and tangential pressures will be sufficient to form initial data set for the collapse. We are going to show the existence of $b_{p}$ 
through $p_{r}$ and $p_{\theta}$. Therefore, we consider $p_{r}, p_{\theta}$ as an initial data set. Using this initial data prescribed at the initial epoch $t=t_{i}$ to evolve the collapse, we can integrate the equation (44) and obtain,

$$
\eta(R)=\int_{0}^{R} \frac{2\left(p_{\theta_{o}}-p_{r_{o}}\right)-R p_{r}^{\prime}}{R\left(\rho_{o}+p_{r_{o}}\right)} d R
$$

Thus, velocity distribution function $\eta(R)$ is determined by smooth functions $p_{r}$ and $p_{\theta}$. Using equations (40) and (43), equation (7) can be written as

$$
\sqrt{R} \dot{R}=-a(t) e^{\eta} \sqrt{\left[1+r^{2} b_{p}\right] R e^{2 \eta}-R+\frac{p_{r}}{3}\left(2 r^{3}-R^{3}\right)}
$$

where $a(t)$ is a function of time and we choose $a(t)=1$ by suitable scaling of time coordinate.

We define

$$
h_{p}(R)=\frac{e^{2 \eta(R)}-1}{R^{2}}=2 g_{p}(R)+O\left(R^{2}\right)
$$

Using this definition, equation (47) takes the form

$$
\sqrt{v} \dot{v}=-\sqrt{v b_{p} e^{4 \eta}+e^{2 \eta}\left[v^{3} h_{p}(r v)+\frac{p_{r}}{3}\left(2-v^{3}\right)\right]}
$$

Integrating above equation, we obtain

$$
t(v, r)=\int_{v}^{1} \frac{\sqrt{v} d v}{\sqrt{v e^{4 \eta} b_{p}+e^{2 \eta}\left[v^{3} h_{p}(r v)+\frac{p_{r}}{3}\left(2-v^{3}\right)\right]}}
$$

where the variable $r$ is treated as a constant. Further, the time taken for the central shell to reach the singularity is given by

$$
t_{s_{o}}=\int_{0}^{1} \frac{\sqrt{v} d v}{\sqrt{v b_{p}(0)+v^{3} h_{p}(0)+\frac{p_{r}}{3}(0)\left(2-v^{3}\right)}}
$$

and $t_{s_{o}}$ is well defined provided $\left[v b_{p}(0)+v^{3} h_{p}(0)+p_{r}(0)\left(2-v^{3}\right) / 3\right]>0$. The time taken for other shells to reach the singularity can be determined from the Taylor expansion of $t(v, r)$ around $r=0$ as $t \rightarrow t_{s}$,

$$
t_{s}(r)=t_{s_{o}}+r \chi_{p}(v)+O\left(r^{2}\right)
$$

where $\chi_{p}(v)=d t(v, r) /\left.d r\right|_{r=0}$ and

$$
\chi_{p}(v)=-\frac{1}{2} \int_{v}^{1} \frac{\sqrt{v}\left[v b_{p}^{\prime}(0)+v^{4} h_{p, r}(0)+\frac{p_{r}^{\prime}(0)}{3}\left(2-v^{3}\right)\right]}{\left[v b_{p}(0)+v^{3} h_{p}(0)+\frac{p_{r}(0)}{3}\left(2-v^{3}\right)\right]^{3 / 2}} d v .
$$

For any constant $\mathrm{v}$ surface, we have $d(v)=0$ which implies $\sqrt{v} \dot{v}=-\left(1 / \chi_{p}(v)\right) \sqrt{v} v^{\prime}$. Now, equation (49) takes the form

$$
\sqrt{v} v^{\prime}=\chi_{p}(v) \sqrt{v b_{p}(0)+v^{3} h(0)+\frac{p_{r}(0)}{3}\left(2-v^{3}\right)}+O\left(r^{2}\right)
$$


The radial null geodesic equation (24) in the variables $\left(u=r^{\alpha}, R\right)$ as $t \rightarrow t_{s}, r \rightarrow 0$, gives

$$
x_{o}^{3 / 2}=\frac{3}{2} \chi_{p}(0) \sqrt{\frac{2}{3} p_{r}(0)}
$$

From equation (53), it is clear that $\chi_{p}(v)$ depends on the functions $b_{p}(0), h_{p}(0), p_{r}(0)$ and these functions, in turn, depend on the given set of density, pressure and velocity function $\nu$. Therefore, equation (55) depicts that tangent to the singularity curve $t=t_{s_{o}}$ is completely determined by the given set of initial data and radial pressure in particular. The radial null geodesic emerging from the singularity in $(R, u)$ co-ordinates is $R=x_{o} u$, while in $(t, r)$ plane, the null geodesic near the singularity is given by $t-t_{s}(0)=x_{o} r^{5 / 3}$.

It is clear that if $\chi_{p}(0)>0$ then $x_{o}>0$ and with this, we get radially outgoing null geodesics coming out from the singularity, giving rise to a naked central singularity. However, no such trajectory comes out if $\chi_{p}(0)<0$ as we have a black hole situation. Thus, we have some region of space-time for which $\chi_{p}(0)>0$ depending on initial data, leading to occurrence of central naked singularity in that space-time region. Therefore, we choose $b_{p}$ to satisfy the following differential equation on a constant $v$-surface

$$
\frac{1}{2} \frac{\sqrt{v}\left[v b_{p}^{\prime}(r)+v^{4} h_{p, r}(r v)+\frac{p_{r}^{\prime}(r)}{3}\left(2-v^{3}\right)\right]}{\left[v b_{p}(r)+v^{3} h_{p}(r v)+\frac{p_{r}(r)}{3}\left(2-v^{3}\right)\right]^{3 / 2}} d v=\mathcal{B}(r, v)
$$

where $\mathcal{B}(r, v)$ is a continuous function defined on $\mathcal{D}$ such that

$$
\frac{1}{2} \frac{\sqrt{v}\left[v b_{p}^{\prime}(0)+v^{4} h_{p, r}(0)+\frac{p_{r}^{\prime}(0)}{3}\left(2-v^{3}\right)\right]}{\left[v b_{p}(0)+v^{3} h_{p}(0)+\frac{p_{r}(0)}{3}\left(2-v^{3}\right)\right]^{3 / 2}} d v=\mathcal{B}(0, v)<0 \quad \text { for all } v \in[0,1] .
$$

It then follows that

$$
\chi_{p}(0)=\lim _{v \rightarrow 0} \chi_{p}(v)=-\int_{0}^{1} \mathcal{B}(0, v) d v>0 .
$$

The above requirement ensures the nakedness of central shell focusing singularity. Now, we discuss existence of $b_{p}$ as a solution of the differential equation (56). For this, we use

$$
y(r, v)=v b_{p}(r)+v^{3} h_{p}(r v)+\frac{p_{r}(r)}{3}\left(2-v^{3}\right)
$$

a continuous function of $r$, in equation (56), and we obtain

$$
\frac{d y}{d r}=\frac{1}{\sqrt{v}}\left[2 \mathcal{B}(r, v) y^{3 / 2}\right] \equiv \varphi(y, r)
$$

with initial condition

$$
y(0, v)=v b_{p}(0)+v^{3} h_{p}(0)+\frac{p_{r}(0)}{3}\left(2-v^{3}\right)
$$

This initial value problem defined throughout the cloud has a $C^{1}$ - function $y(r, v)$ as its unique solution defined over the entire cloud. Using the arguments of general case (section III) for the function $\varphi(y, r)$ and $\mathcal{B}(r, v))$ a weaker condition

$$
0 \leq|\mathcal{B}(r, v)| y^{3 / 2} \leq \frac{n \sqrt{v}}{2 r_{c}}
$$


holds for all $r$ in $\left[0, r_{c}\right]$ and $v \in[0,1]$ where $n$ is a finite number such that $0 \leq y \leq n$. This condition holds true for $y$ restricted to a bounded domain and the collapse may start with $r_{c}$ small enough. Therefore, there are infinitely many choices for the function $\mathcal{B}(r, v)$, which is continuous and satisfies the conditions (57) and (62) for each choice of $v$. For each such $\mathcal{B}(r, v)$, there will be a unique solution $y(r, v)$ of the differential equation $(60)$, satisfying initial condition (61), defined over the entire cloud. This unique solution $y(r, v)$ for each such choice of $\mathcal{B}(r, v)$ yields

$$
b_{p}=\frac{y(r, v)-v^{3} h_{p}(r v)-\frac{p_{r}(r)}{3}\left(2-v^{3}\right)}{v}
$$

over $\left[0, r_{c}\right]$. As each function appearing in the above expression is continuous, therefore, the condition holds true as $v \rightarrow 0$, because of continuity. We know that velocity distribution function $\eta(R)$ is determined through smooth functions $p_{r}, p_{\theta}$ on a given constant $v$-surface, which in turn, through equation (48) determines the function $h_{p}(r v)$. Thus, for a given constant $v$-surface and given initial data of radial and tangential pressures $p_{r}$ and $p_{\theta}$, there exists infinitely many choices of the function $b_{p}$ such that condition (57) is satisfied. We have thus proved the existence of energy distribution function $b_{p}$ for a given set of initial data leading to the occurrence of a naked central shell focusing singularity.

Now, we illustrate the stability of occurrence of NS. For simplicity, we consider the evolution of initial data on the initial surface $t=t_{i}$ where $v=1$ and $v^{\prime}=0$. Using equations (42) and (45), the energy conditions $\rho \geq 0 ; \rho+p_{r} \geq 0 ; \rho+p_{\theta} \geq 0$ can be described as

$$
\begin{array}{r}
\rho=p_{r}+\frac{1}{3} r p_{r}^{\prime} \equiv K_{1} \geq 0, \quad 2 p_{r}+\frac{1}{3} r p_{r}^{\prime} \equiv K_{2} \geq 0, \\
2\left(2+r \eta_{, R}\right) p_{r}+\left[1+\frac{1}{3}\left(2+r \eta_{, R}\right)\right] r p_{r}^{\prime} \equiv K_{3} \geq 0
\end{array}
$$

We denote by $X_{p}$ be an infinite dimensional Banach space of all $C^{1}$ real-valued functions defined on $\left[0, r_{c}\right]$, endowed with the $C^{1}$ norm. Let us take $G_{p}=\left\{p_{r}(r): p_{r}>0, p_{r}\right.$ is $C^{1}, K_{1}>0$ and $K_{3}>0$ on $\left.\left[0, r_{c}\right]\right\}$ be a subset of $X_{p}$.

Now, to show that $G_{p}$ is an open set in $X_{p}$. For $p_{r}$ in $G_{p}$, we put $\alpha_{1}=\min \left(p_{r}\right)$, $\alpha_{2}=\min \left(K_{1}\right), \alpha_{3}=\min \left(K_{3}\right)$, and $\lambda_{p}=\max \left(2+r \eta_{, R}\right)$. We define a positive real number

$$
\mu=\frac{1}{2} \min \left\{\alpha_{1}, \frac{\alpha_{2}}{3}, \frac{\alpha_{2}}{r_{c}}, \frac{\alpha_{3}}{6 \lambda_{p}}, \frac{\alpha_{3}}{3 r_{c}}, \frac{\alpha_{3}}{3 r_{c} \lambda_{p}}\right\}
$$

Let $p_{r_{1}}(r)$ be $C^{1}$ in $\left[0, r_{c}\right]$ with $\left\|p_{r}-p_{r_{1}}\right\|<\mu$. Now, using definition of standard $C^{1}$ norm, we get $\left|p_{r}-p_{r_{1}}\right|<\mu$ and $\left|p_{r}^{\prime}-p_{r_{1}}^{\prime}\right|<\mu$ over $\left[0, r_{c}\right]$. Then, for each choice of $\mu$, the respective inequalities

$$
\begin{aligned}
p_{r_{1}}>p_{r}-\frac{\alpha_{1}}{2}>0, \quad\left|p_{r_{1}}-p_{r}\right|<\frac{\alpha_{2}}{6}, \frac{1}{3} r\left|p_{r_{1}^{\prime}}-p_{r}^{\prime}\right| \leq \frac{1}{3} r_{c}\left|p_{r_{1}}^{\prime}-p_{r}^{\prime}\right|<\frac{\alpha_{2}}{6}, \\
2\left(2+r \eta_{, R}\right)\left|p_{r_{1}}-p_{r}\right| \leq 2 \lambda_{p}\left|p_{r_{1}}-p_{r}\right|<\frac{\alpha_{3}}{6}, r\left|p_{r_{1}^{\prime}}-p_{r}^{\prime}\right| \leq r_{c}\left|p_{r_{1}}^{\prime}-p_{r}^{\prime}\right|<\frac{\alpha_{3}}{6} \\
\frac{r}{3}\left(2+r \eta_{, R}\right)\left|p_{r_{1}}^{\prime}-p_{r}^{\prime}\right| \leq r_{c} \lambda_{p}\left|p_{r_{1}}^{\prime}-p_{r}^{\prime}\right|<\frac{\alpha_{3}}{6}
\end{aligned}
$$


are satisfied in $\left[0, r_{c}\right]$. Using above inequalities analogous to the general case, we obtain $p_{r_{1}}>0,\left[p_{r_{1}}+r p_{r_{1}}^{\prime} / 3\right]>0, \quad\left[2\left(2+r \eta_{, R}\right) p_{r_{1}}+\left[1+\left(2+r \eta_{, R}\right) / 3\right] r p_{r_{1}}^{\prime}\right]>0$, provided $\left(2+r \eta_{, R}\right)>0$ on $\left[0, r_{c}\right]$. Therefore, $p_{r_{1}} \in G_{p}$ and hence, $G_{p}$ is an open set in $X_{p}$.

The function $\eta(R)$ is determined by $p_{r}, p_{\theta}$ on the given constant $v$-surface and the condition $\left(2+r \eta_{, R}\right)>0$ is necessary for openness of the set $G_{p}$. Therefore, the $C^{1}$ function $h_{p}(R)=\left(e^{2 \eta(R)}-1\right) / R^{2}$ is also determined by initial data $p_{r}, p_{\theta}$ on a given constant $v$-surface constrained with $\left(2+r \eta_{, R}\right)>0$ on $\left[0, r_{c}\right]$. Let $\mathcal{H}_{p}$ be a subspace of $X_{p}$ containing such initial data $h_{p}(R)$. Then $G_{p} \times \mathcal{H}_{p}$ is an open set in the product space $X_{p} \times X_{p}$.

Let $\left(p_{r_{1}}, h_{p_{1}}(r)\right)$ be in the neighbourhood of $\left(p_{r}, h_{p}(r)\right)$ in $G_{p} \times \mathcal{H}_{p}$. Using equation (62) analogously for $p_{r_{1}}$ and $h_{p_{1}}(r)$, we have a choice of infinitely many $\mathcal{B}_{1}(r, 1)$, such that for each such $\mathcal{B}_{1}$, there will exist a unique $b_{1}(r)$ so that the initial data $p_{r_{1}}$ and $h_{p_{1}}(r)$ together will lead the collapse to formation of a naked singularity. Thus, naked singularity arising from initial data $\left(p_{r}, h_{p}(r)\right)$ is $C^{1}$ - stable. Thus, this particular case illustrates clearly how the choice of initial data leads the collapse to a naked singularity and how it is stable under small perturbations of initial data in an appropriate mathematical sense.

\section{Discussion and conclusions}

1. Our main conclusions in the paper are the following: (a) Given a $C^{2}$ - mass function $M(r, v)$ and a $C^{1}$ - function $h(r, v)$, on any $\mathrm{v}=$ constant surface, we find a $C^{1}$ - energy distribution function $b(r)$ such that the collapse ends in a naked singularity. (b) With physically reasonable conditions put on mass function $M$, and with $C^{1}$ - function $h(r, v)$, the initial data consisting of $(M, h(r, v))$ leading the collapse to a naked singularity forms an open subset of the space $X \times Y$. This establishes stability of naked singularity with respect to initial data.

2. Similar analysis for the case $\chi(0)<0$ ( that is $B(0, v)>0$ for all $v$ in $[0,1]$ ) shows that the set $G \times \mathcal{H}$ of 'true' initial data $(M, h(r, v))$, leading the collapse to a black hole forms an open set and it is neither dense nor nowhere dense in the space of all initial data. Thus, occurrence of black hole is stable but not generic.

3. Our analysis is carried out with given initial data on a constant $v$ - surface. Hence, the role of $M$ and $b(r)$ can be interchanged (refer equation 35) such that the mass function would evolve in the continuous gravitational collapse.

4. The conditions $w>0$ and $w_{1}>0$ which occurred in our stability analysis of NS (general case) can yield some information about the function $\nu$. For simplicity, let us consider these conditions on the initial surface $t=t_{i}$. For $w>0$, we obtain $\nu_{, r}>-2 / r$ for all $r \in\left[0, r_{c}\right]$. This means $\nu_{, r} \in\left(-2 / r_{c}, \infty\right)$. Next, for $w_{1}>0$, we get $\left[-(2+r) \nu_{, r}\right]>0$, this implies $\nu_{, r} \in(-\infty, 0)$. Thus, common domain of $\nu_{, r}$ is $\left(-2 / r_{c}, 0\right)$. Therefore, $\nu$ is a decreasing function of $r$ in $\left[0, r_{c}\right]$ for sufficiently small $r_{c}$.

While, in particular case $\left[2+r \eta_{, R}\right]>0$ yields $\eta_{, r} \in\left(-2 / r_{c}, \infty\right)$, therefore, $\eta(r)$ is a decreasing function of $r$ in $\left[0, r_{c}\right]$. Also, the above said condition can be written as $\left[6\left(p_{r}+p_{\theta}\right)-r p_{r}^{\prime}\right]>0$, it means $C^{1}$ - stability of occurrence of NS is associated with initial data $p_{r}$ and $p_{\theta}$ such that $\left[6\left(p_{r}+p_{\theta}\right)-r p_{r}^{\prime}\right]>0$. It is this choice of data that gives rise to stable NS.

5. The space $X$ used in the analysis is an infinite-dimensional Banach space, and as such, is not locally compact. It is not therefore, straightforward to define a measure on 
this space. Hence, we can not say whether the set $\mathcal{G}$ is of zero measure or not. However, as mentioned earlier, $\mathcal{G}$ is not meagre, and thus, it is a substantially 'big' set. Similar argument is applicable to the set $\mathcal{G} \times \mathcal{H}$ in the product space $X \times Y$. We believe that rigorous analysis that we have worked out shall help further in understanding the gravitational collapse of Type I matter fields.

\section{Acknowledgement:}

We register our deep gratitude to Dr. S. H. Ghate for his valuable guidance. One of the authors (SBS) would like to thank R. Goswami for many helpful suggestions.

\section{References}

[1] P. S. Joshi and I. H. Dwivedi, Class. Quantum Grav. 16 (1999) 41-59

[2] P. S. Joshi, Pramana, A jour. of physics (2000) pp 1-16 , gr-qc/0006101.

[3] P. S. Joshi and R. Goswami, Physical Review D 69, 064027 (2004)

[4] R. Penrose, Riv. Nuovo Cimento 1, 252 (1969); in General Relativity, an Einstein Centenary Volume, edited by S. W. Hawking and W. Israel (Cambridge University Press, Cambridge, England, 1979).

[5] F. I. Cooperstock, S. Jhingan, P. S. Joshi and T. P. Singh, Class. Quantum Grav. 14 (1997) 2195-2201

[6] S. W. Hawking and G. F. R. Ellis, The Large Scale Structure of Space-time (Cambridge University Press, Cambridge, 1973).

[7] P. S. Joshi, Global Aspects in Gravitation and Cosmology (Clarendon Press, Oxford, 1993)

[8] R. Goswami and P. S. Joshi, gr-qc/0406052, (To appear in Class. Quantum Grav.)

[9] I. H. Dwivedi and P. S. Joshi, Commun. Math. Phys. 166 (1994) 117-128.

[10] F. Verhulst Non-linear Differential Equations and Dynamical Systems (Berlin: Springer)

[11] R. Abraham and J. Marsden, Foundations of Mechanics, 2nd edition (The Benjamin/Cummings Publishing Company, 1978)

[12] R. V. Saraykar and S. H. Ghate, Class. Quantum Grav. 16 (1999) 281-289.

[13] R. Goswami and P. S. Joshi, Class. Quantum Grav. 19 (2002) 5229-5234. 